\title{
RADIUS PROBLEMS FOR A SUBCLASS OF CLOSE-TO-CONVEX UNIVALENT FUNCTIONS
}

\author{
KHALIDA INAYAT NOOR \\ Mathematics Department \\ College of Science \\ King Saud University, Riyadh 11451 \\ Saudi Arabia
}

(Received July 16, 1990 and in revised form December 26, 1990)

ABSTRACT. Let $P[A, B],-1 \leq B<A \leq 1$, be the class of functions $p$ such that $p(z)$ is subordinate to $\frac{1+A z}{1+B z}$. A function $f$, analytic in the unit disk $E$ is said to belong to the class $K_{\beta}^{*}[A, B]$ if, and only if, there exists a function $g$ with $\frac{z g^{\prime}(z)}{g(z)} \in P[A, B]$ such that $\operatorname{Re} \frac{\left(z f^{\prime}(z)\right)^{\prime}}{g^{\prime}(z)}>\beta$, $0 \leq \beta<1$ and $z \in E$. The functions in this class are close-to-convex and hence univalent. We study its relationship with some of the other subclasses of univalent functions. Some radius problems are also solved.

KEY WORDS AND PHRASES. Close-to-convex, starlike univalent, convex, radius of convexity. 1991 AMS SUBJECT CLASSIFICATION CODE. 30A32, 30A34.

1. INTRODUCTION.

Let $f$ be analytic in $E=\{z:|z|<1\}$ and be given by

$$
f(z)=z+\sum_{n=2}^{\infty} a_{n} z^{n}
$$

A function $g$, analytic in $E$, is called subordinate to a function $G$ if there exists a Schwarz function $w(z)$, analytic in $E$ with $w(0)=0$ and $|w(z)|<1$ in $E$, such that $g(z)=G(w(z))$.

In [1], Janowski introduced the class $P[A, B]$. For $A$ and $B,-1 \leq B<A \leq 1$, a function $p$, analytic in $E$ with $p(0)=1$ belongs to the class $P[A, B]$ if $p(z)$ is subordinate to $\frac{1+A z}{1+B z}$. When $A=1, B=-1$, we obtain the class $P$ of functions with positive real part in $E$. Also for $A=1-2 \beta, B=-1,0 \leq \beta<1$, we have the class $P(\beta)$. A function $h \in P(\beta), 0 \leq \beta<1$ if and only if $\operatorname{Re} h(z)>\beta, z \in E$.

Let $S^{*}[A, B]$ and $C[A, B]$ denote the classes of functions, analytic in $E$, and given by (1.1) such that $\frac{z f^{\prime}(z)}{f(z)} \in P[A, B]$ and $\frac{\left(z f^{\prime}(z)\right)^{\prime}}{f^{\prime}(z)} \in P[A, B]$ respectively. Also, for $B=-1$ and $A=1-2 \gamma$, $0 \leq \gamma<1$, we have $S^{*}(\gamma)$ and $C(\gamma)$ the classes of starlike and convex functions of order $\gamma$, see [2].

Now we have the following: 
DEFINITION 1.1: Let $f$ be analytic in $E$ and be given by (1.1). Then $f$ is said to be in the class $K_{\beta}[A, B],-1 \leq B<A \leq 1$ if and only if there exists a $g \in S^{*}[A, B]$ such that, for $z \in E$. $\frac{z f^{\prime}(z)}{g(z)} \in P(\beta)$.

This class has been defined and studied by Silvia [3] in a more general way. W'hen $B=-1, A=1$ and $\beta=0$, we have the class $K$ of close-to-convex univalent functions.

DEFINITION 1.2.: Let $f$ be analytic in $E$ and be given by (1.1). Then $f \varepsilon K_{\beta}^{*}[A, B]$ if and only there exists a $g \in S^{*}[A, B]$ such that $\frac{\left(z f^{\prime}(z)\right)^{\prime}}{g^{\prime}(z)} \in P(\beta)$ for $z \in E$.

For $\beta=0, A=1$ and $B=-1$, we obtain the class $K^{*}$ discussed in [4].

If we take $g \in C[A, B]$ in Definition 1.2, we obtain the class $C_{\beta}^{*}[A, B]$. The special cases of this class have been investigated in $[5,6,7]$.

We shall focus on the class $K_{\beta}^{*}[A, B]$ and establish the relationship of this class with some other subclasses of close-to-convex functions. It is clear that

and

$$
C[A, B] \subset S^{*}[A, B] \subset K_{\beta}[A, B] \subset K
$$

$$
C[A, B] \subset C_{\beta}^{*}[A, B] \subset K_{\beta}^{*}[A, B] \subset K_{\beta}[A, B] \subset K
$$

We shall also solve some radius problems for the functions in $K_{\beta}^{*}[A, B]$.

2. PRELIMINARY RESULTS.

We shall need the following:

LEMMA 2.1 [8]: If $f \in C(\gamma)$, then $f(z)$ is analytic, univalent and starlike of order $\lambda(\gamma)$ where, for $0 \leq \gamma<1$,

$$
\lambda(\gamma)=\left\{\begin{array}{cc}
\frac{4^{\gamma}(1-2 \gamma)}{4-2^{2 \gamma+1},} & \gamma \neq \frac{1}{2} \\
(\log 4)^{-1}, & \gamma=\frac{1}{2}
\end{array}\right.
$$

This result is sharp.

LEMMA 2.2. Let $p \in P(\beta), 0 \leq \beta<1$. Then

i) $\quad p(z)=(1-\beta) h(z)+\beta, h \in P \quad$ (see [2]).

ii) $\left|p^{\prime}(z)\right| \leq \frac{2[\operatorname{Re} p(z)-\beta]}{1-r^{2}}$

iii) $\left|\frac{p^{\prime}(z)}{p(z)}\right| \leq \frac{2(1-\beta)}{(1-r)((1-2 \beta) r+1)}$

For (ii) and (iii), we refer to [9].

LEMMA 2.3. The radius of convexity of $S^{*}[A, B]$ is given by the smallest root $r_{o}$ in $(0,1)$ of

i) $\quad A^{2} r^{2}-(3 A-B) r+1=0 \quad$ if $R_{1} \leq R_{2}$

ii) $[(A-B)+4 A(1-A)] r^{4}+2\left[(A-B)+2(1-A)^{2}\right] r^{2}+(A-B) r-4(1-A)=0, \quad$ if $R_{2} \leq R_{1}$, 
where

and

$$
R_{1}=\left(\frac{L}{K}\right)^{1 / 2}, \quad R_{2}=\frac{1-A r}{1-B r}, \quad L=(1-A)\left(1+A r^{2}\right)
$$

$$
K=(A-B)\left(1-r^{2}\right)+(1-B)\left(1+B r^{2}\right) .
$$

LEMMA 2.4. Let $p \in P[A, B]$. Then

$$
\frac{1-A r}{1-\overline{B r}} \leq \operatorname{Re} p(z) \leq|p(z)| \leq \frac{1+A r}{1+B r}
$$

LEMMA 2.5. Let $N$ and $D$ be analytic in $E, D$ maps onto a many-sheeted starlike region. $N(0)=0=D(0)$ and $\frac{N^{\prime}(z)}{D^{\prime}(z)} \in P[A, B]$. Then $\frac{N(z)}{D(z)} \in[A, B]$.

For the above two lemmas we refer to [11].

\section{MAIN RESULTS.}

From Definition 1.2 and Lemma 2.5 , we clearly see that the function $f$ belonging to $K_{\beta}^{*}[A, B]$ is close-to-convex and hence univalent. In fact, we can prove the following:

THEOREM 3.1. Let $f \in K_{\beta}^{*}[A, B], 0 \leq \beta<1$. Then $f \in K_{\sigma}[A, B]$, where $\sigma(\beta)$ is given as

$$
\sigma(\beta)=\left\{\begin{array}{cc}
\frac{4^{\beta}(1-2 \beta)}{4-2^{2 \beta+1},} & \beta \neq \frac{1}{2} \\
(\log 4)^{-1}, & \beta=\frac{1}{2}
\end{array}\right.
$$

This result is sharp for $A=1-b \beta, \beta=1$.

PROOF. Since $f \in K_{\beta}^{*}[A, B]$, there exists a $g \in S^{*}[A, B]$ such that, for $z \in E$,

$$
\begin{aligned}
\frac{\left(z f^{\prime}(z)\right)^{\prime}}{g^{\prime}(z)} & =(1-\beta) h(z)+\beta, \quad h \in P \\
& =(1-\beta) \frac{z \phi^{\prime}(z)}{\phi(z)}+\beta, \quad \text { for some } \phi \in S^{*} \\
& =\frac{N^{\prime}(z)}{D^{\prime}(z)}
\end{aligned}
$$

So

$$
\begin{aligned}
\frac{N(z)}{D(z)}=\frac{z f^{\prime}(z)}{g(z)} & =\frac{z\left(\frac{\phi(z)}{z}\right)^{1-\beta}}{\int_{0}^{z}\left(\frac{\phi(t)}{t}\right)^{1-\beta} d t} \\
& =\frac{1}{\int_{0}^{z}\left(\frac{z}{t}\right)^{1-\beta}\left[\frac{\phi(t)}{\phi(z)}\right]^{1-\beta} \frac{d t}{z}}
\end{aligned}
$$

where we integrate along the straight line segment $[0,2], z \in E$. Using Lemma 2.5 for $B=-1$ and 
$A=1-2 \beta$, we conclude that $\operatorname{Re} \frac{N(z)}{D(z)}=\operatorname{Re} \frac{z f^{\prime}(z)}{g(z)}>\beta \geq 0$, and since $\frac{z f^{\prime}(z)}{g(z)}=1$ at $z=0$, we have

$$
\left|\frac{z f^{\prime}(z)}{g(z)}-\frac{1+r^{2}}{1-r^{2}}\right| \leq \frac{2 r}{1-r^{2}}
$$

$|z|=r, \quad z \in E$; see [12].

From (3.4) it is clear that

$$
\begin{aligned}
& \underset{f \in K_{\beta}^{*}[A, B]}{\operatorname{Min}} \underset{|z|=r}{\operatorname{Min}} \operatorname{Re} \frac{z f^{\prime}(z)}{g(z)} \\
& =\underset{f \in K_{\beta}^{*}[A, B]}{\operatorname{Min}}|\underset{z i n}{=}| \frac{z f^{\prime}(z)}{g(z)} \mid,
\end{aligned}
$$

and hence it is sufficient to find the minimum of the right hand side of (3.3). Then from [8], we have

$$
\sigma(\beta)=\min \left[\llbracket \int_{0}^{z}\left(\frac{z}{t}\right)^{1-\beta}\left(\frac{\phi(t)}{\phi(z)}\right)^{1-\beta} \frac{d t}{z} \|\right]^{-1},
$$

for $\phi \in S^{*}, z \in E$ and $\sigma(\beta)$ is as given in (3.1). This proves our result.

Sharpness for $A=1-2 \beta, B=1$ follows by taking

$$
f_{\beta}(z)=g_{\beta}(z)= \begin{cases}\frac{1-(1-z)^{2 \beta-1}}{2 \beta-1}, & \beta \neq \frac{1}{2} \\ \log (1-z)^{-1}, & \beta=\frac{1}{2}\end{cases}
$$

Using Definition 1.2 and Lemma 2.1, we immediately have the following:

THEOREM 3.2. Let $f \in C_{\beta}^{*}[1-2 \gamma,-1]$. Then $f \in K_{\beta}^{*}[1-2 \lambda, 1]$, where $\lambda(\gamma)$ is as given in Lemma 2.1.

THEOREM 3.3. Let $f \in K_{\beta}^{*}[A, B]$. Then there exists a $g \in C[A, B]$ such that $h$ defined by

$$
h^{\prime}(z)=\frac{\left(z f^{\prime}(z)\right)^{\prime}}{1+\frac{z g^{\prime \prime}(z)}{g^{\prime}(z)}}
$$

belongs to $K_{\beta}[A, B]$, for $z \in E$.

PROOF. Since $f \in K_{\beta}^{*}[A, B]$, we have $\frac{\left(z f^{\prime}(z)\right)^{\prime}}{G^{\prime}(z)} \in P(\beta), G \in S^{*}[A, B]$. Let $G(z)=z g^{\prime}(z)$, so $g \in C[A, B]$. Now

Thus

$$
G^{\prime}(z)=\left(z g^{\prime}(z)\right)^{\prime}=g^{\prime}(z)\left[1+\frac{z g^{\prime \prime}(z)}{g^{\prime}(z)}\right]
$$

$$
\frac{\left(z f^{\prime}(z)\right)^{\prime}}{G^{\prime}(z)}=\frac{\left(z f^{\prime}(z)\right)^{\prime}}{g^{\prime}(z)\left[1+\frac{z g^{\prime \prime}(z)}{g^{\prime}(z)}\right]}=\frac{h^{\prime}(z)}{g^{\prime}(z)}
$$

and this implies $h \in K_{\beta}[A, B]$.

We now deal with the radius problems. 
THEOREM 3.4. Let $f \in K_{\beta}[A, B], z \in E$. Then $f \in K_{\beta}^{*}[A, B]$ for $|z|<r_{1}$, where $r_{1}$ is the least positive root in $(0,1)$ of the equation

$$
1-(A+2) r+(2 B-1) r^{2}+A r^{3}=0
$$

PROOF. For $z \in E$, we can write

$$
z f^{\prime}(z)=g(z) h(z), \quad h \in P(\beta) \text { and } g \in S^{*}[A, B] .
$$

Then

$$
\frac{\left(z f^{\prime}(z)\right)^{\prime}}{g^{\prime}(z)}=h(z)+\frac{g(z)}{g^{\prime}(z)} h^{\prime}(z)
$$

from which it follows that

$$
\operatorname{Re}\left[\frac{\left(z f^{\prime}(z)\right)^{\prime}}{g^{\prime}(z)}-\beta\right] \geq \operatorname{Re} h(z)-\beta-\left|\frac{g(z)}{g^{\prime}(z)} h^{\prime}(z)\right| .
$$

Now, since $g \in S^{*}[A, B]$, it follows from Lemma 2.4 that

$$
\left|\frac{g(z)}{g^{\prime}(z)}\right| \leq \frac{r(1-B r)}{1-A r}
$$

Using (3.5) and Lemma 2.2(ii) we have

$$
\begin{aligned}
\operatorname{Re}\left[\frac{\left(z f^{\prime}(z)\right)^{\prime}}{g^{\prime}(z)}-\beta\right] & \geq[\operatorname{Re} h(z)-\beta]\left\{1-\frac{2 r}{1-r^{2}} \frac{1-B r}{1-A r}\right\} \\
& =[\operatorname{Re} h(z)-\beta]\left[\frac{1-(A+2) r+(2 B-1) r^{2}+A r^{3}}{\left(1-r^{2}\right)(1-A r)}\right]
\end{aligned}
$$

and this gives us the required result.

THEOREM 3.5. Let $f \in K_{\beta}^{*}[A, B]$. Then $f \in C_{\beta}^{*}[1,-1]$ for $|z|<r_{o}$, where $r_{o}$ is as given in Lemma 2.3.

PROOF. Since $f \in K_{\beta}^{*}[A, B]$ implies that $\frac{\left(z f^{\prime}(z)\right)^{\prime}}{g^{\prime}(z)} \in P(\beta), g \in S^{*}[A, B], z \in E$. To show that $f \in C_{\beta}^{*}[1,-1]$ for $|z|<r_{o}$, it is sufficient to prove that $g \in C[1,-1] \equiv C$ for $|z|<r_{o}$ and this follows immediately from Lemma 2.3 . Hence the theorem.

THEOREM 3.6. Let $F=z f^{\prime}$ and let $f \in K_{\beta}^{*}[A, B]$. Then $F$ maps $|z|<r_{2}$ onto a convex domain, where $r_{2}$ is the least positive root in $(0,1)$ of the equation

$$
(1-2 \beta) r^{3}+\left(r_{o}+2\right)(2 \beta-1) r^{2}-\left(2 r_{o}+1\right) r+r_{o}=0
$$

and $r_{o}$ as given in Lemma 2.3. 
PROOF. $z F^{\prime}(z)=z\left(z f^{\prime}(z)\right)^{\prime}=z g^{\prime}(z) h(z), \quad h \in P(\beta), g \in S^{*}[A, B]$ Thus

$$
\frac{\left(z F^{\prime}(z)\right)^{\prime}}{F^{\prime}(z)}=\frac{\left(z g^{\prime}(z)\right)^{\prime}}{g^{\prime}(z)}+\frac{z h^{\prime}(z)}{h(z)}
$$

and

$$
\operatorname{Re} \frac{\left(z F^{\prime}(z)\right)^{\prime}}{F^{\prime}(z)} \geq \operatorname{Re} \frac{\left(z g^{\prime}(z)\right)^{\prime}}{g^{\prime}(z)}-\left|\frac{z h^{\prime}(z)}{h(z)}\right|
$$

Since $g \in S^{*}[A, B]$, it follows from Lemma 2.3 that $g \in C[1,-1] \equiv C$ for $|z|<r_{o}$. So we have, see [12],

$$
\operatorname{Re} \frac{\left(z g^{\prime}(z)\right)^{\prime}}{g^{\prime}(z)} \geq \frac{r_{o}-r}{r_{o}+r}
$$

Using (3.6) and Lemma 2.2(iii), we have

$$
\begin{aligned}
\operatorname{Re} \frac{\left(z F^{\prime}(z)\right)^{\prime}}{F^{\prime}(z)} & \geq \frac{r_{o}-r}{r_{o}+r}-\frac{2 r(1-\beta)}{(1-r)((1-2 \beta) r+1)} \\
& =\frac{\left(r_{o}-r\right)(1-r)((1-2 \beta) r+1)-2 r(1-\beta)\left(r_{o}+r\right)}{\left(r_{o}+r\right)(1-r)((1-2 \beta) r+1)}
\end{aligned}
$$

After simplification we obtain the required result.

THEOREM 3.7. Let $F \in K_{\beta}^{*}[A, B]$ with respect to $G \in S^{*}[A, B], 0 \leq \beta<1$. Let, for $0<\alpha \leq \frac{1}{2}$,

and

$$
f(z)=(1-\alpha) F(z)+\alpha z F^{\prime}(z),
$$

$$
g(z)=(1-\alpha) G(z)+\alpha z G^{\prime}(z)
$$

Then $f \in K_{\beta}^{*}[A, B]$ with respect to $g$ for $|z|<r$, where $r=\min \left(r_{4}, r_{3}\right) \quad$ with $r_{4}=\frac{1}{2 \alpha+\sqrt{4 \alpha^{2}-2 \alpha+1}}$ and $r_{3}$ the least positive root in $(0,1)$ of the equation

$$
r_{o}+\left[1-2 \alpha\left(1+r_{o}\right)\right] r-\left(r_{o}+2 \alpha\right) r^{2}-(1-2 \alpha) r^{3}=0
$$

The number $r_{o} \in(0,1)$ is given in Lemma 2.3.

PROOF. We can write (3.7) as

$$
F(z)=\frac{1}{\alpha} z^{1-\frac{1}{\alpha}} \int_{0}^{z} z^{\frac{1}{\alpha}-2} f(z) d z
$$


So

$$
\begin{aligned}
z F^{\prime}(z) & =\frac{1}{\alpha} z^{1-\frac{1}{\alpha}}\left[\left(1-\frac{1}{\alpha}\right) \int_{0}^{z} z^{\frac{1}{\alpha}-2} f(z) d z+z^{\frac{1}{\alpha}-1} f(z)\right] \\
& =\frac{1}{\alpha} z^{1-\frac{1}{\alpha}}\left[\int_{0}^{z} z^{\frac{1}{\alpha}-1} f^{\prime}(z) d z\right]
\end{aligned}
$$

Thus

$$
\begin{aligned}
\frac{\left(a F^{\prime}(z)\right)^{\prime}}{G^{\prime}(z)} & =\frac{z^{\frac{1}{\alpha}} f(z)-\left(\frac{1}{\alpha}-1\right) \int_{0}^{z} z^{\frac{1}{\alpha}-1} f^{\prime}(z) d z}{\left(\frac{1}{\alpha}-1\right) \int_{0}^{z} z^{\frac{1}{\alpha}-1} g^{\prime}(z) d z} \\
& =(1-\beta) h(z)+\beta, \quad h \in P .
\end{aligned}
$$

Differentiating both sides and simplifying, we obtain

Now

$$
\operatorname{Re}\left[\frac{\left(z f^{\prime}(z)\right)^{\prime}}{g^{\prime}(z)}-\beta\right] \geq(1-\beta) \operatorname{Re} h(z)\left[1-\frac{2}{1-r^{2}}\left|\frac{\int_{0}^{z} z^{\frac{1}{\alpha}-1} g^{\prime}(z) d z}{z^{\frac{1}{\alpha}-1} g^{\prime}(z)}\right|\right]
$$

$$
\frac{z^{\frac{1}{\alpha}-1} g^{\prime}(z)}{\int_{0}^{z} z^{\left(\frac{1}{\alpha}-1\right)} g^{\prime}(z) d z}=\left(\frac{1}{\alpha}-1\right)+\frac{\left(z G^{\prime}(z)\right)^{\prime}}{G^{\prime}(z)}
$$

Using (3.6) and (3.11), the relation (3.10) yields

$$
\begin{gathered}
\operatorname{Re}\left[\frac{\left(z f^{\prime}(z)\right)^{\prime}}{g^{\prime}(z)}-\beta\right] \geq(1-\beta) \operatorname{Re} h(z)\left[1-\frac{2}{1-r^{2}} \frac{\alpha r\left(r_{o}+r\right)}{r_{o}+(1-2 \alpha) r}\right] \\
=(1-\beta) \operatorname{Re} h(z)\left[\frac{r_{o}\left(1-2 \alpha-2 \alpha r_{o}\right) r-\left(r_{o}+2 \alpha\right) r^{2}-(1-2 \alpha) r^{3}}{\left(1-r^{2}\right)\left[r_{o}+(1-2 \alpha) r\right]}\right]
\end{gathered}
$$

Since it is known [13] that $g \in S^{*}[A, B]$ for $|z|<r_{4}=\frac{1}{2 \alpha+\sqrt{4 \alpha^{2}-2 \alpha+1}}$, we obtain from (3.12) that $f \in K_{\beta}^{*}[A, B]$ for $|z|<r=\min \left(r_{4}, r_{3}\right)$, where $r_{3}$ is the least positive root of (3.9).

ACKNOWLEDGEMENT. The author is grateful to the referee for his helpful suggestions and comments.

\section{REFERENCES}

1. JANOWSKI, J., Some extreme problems for certain families of analytic functions, Ann. Polon. Math. 28(1973), 297-326.

2. ROBERTSON, M.S., On the theory of univalent functions, Ann. Math. 37(1936), 374-408.

3. SILVIA, E.M., Subclasses of close-to-convex functions, Inter. J. Math. \& Math. Sci. 6(1983), 449-458.

4. NOOR, K.I. and AL-DIHAN, N., A subclass of close-to-convex functions, Pb. Univ. J. Math. 16(1982), 183-192. 
5. NOOR, K.I. and THOMAS, D.K., On quasi-convex univalent functions, Inter. J. Math. \& Math. Sci. 3(1980) 255-266.

6. NOOR, K.I., On a subclass of close-to-convex functions, Comm. Math. Univ. St. Pauli, $\underline{29}(1980), 25-28$.

7. NOOR, K.I., On quasi-convex functions and related topics, Inter. J. Math. \& Math. Sci. 10(1987), 241-258.

8. GOEL, R.M., Functions starlike and convex of order $\alpha$, J. London Math. Soc. 2(1974), 128-130.

9. McCARTY, C.P., Functions with real part greater than $\alpha$, Proc. Amer. Math. Soc. 35(1972), 211-216.

10. ANH, V.V., K-fold symmetric starlike univalent functions, Bull. Austral. Math. Soc. 32(1985), 419-436.

11. PARVATHAM, R. and SHANMUGHAM, T.N., On analytic functions with reference to an integral operator, Bull. Austral. Math. Soc. 28(1983), 207-215.

12. NEHARI, Z., Conformal Mapping, McGraw-Hill, New York, 1954.

13. NOOR, K.I., On some subclasses of close-to-convex functions in Univalent Functions, Fractional Calculus and Their Applications, ed. by H. Srivistava and S. Owa, J. Wiley and Sons London, 1989. 


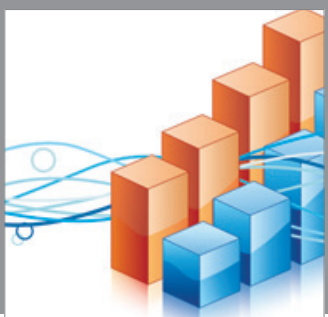

Advances in

Operations Research

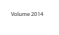

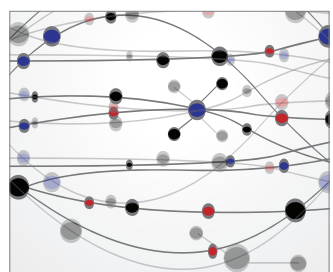

\section{The Scientific} World Journal
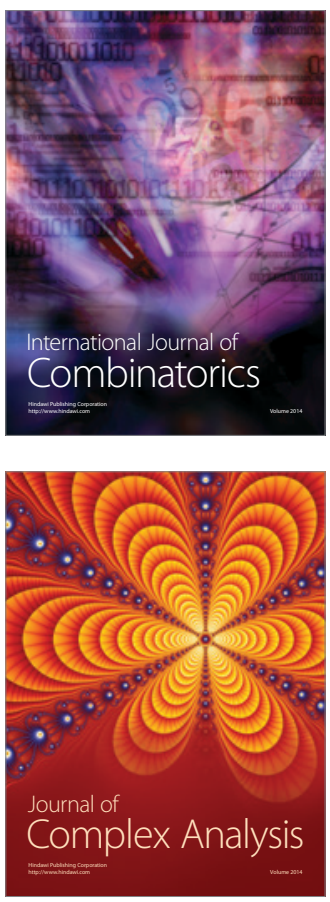

International Journal of

Mathematics and

Mathematical

Sciences
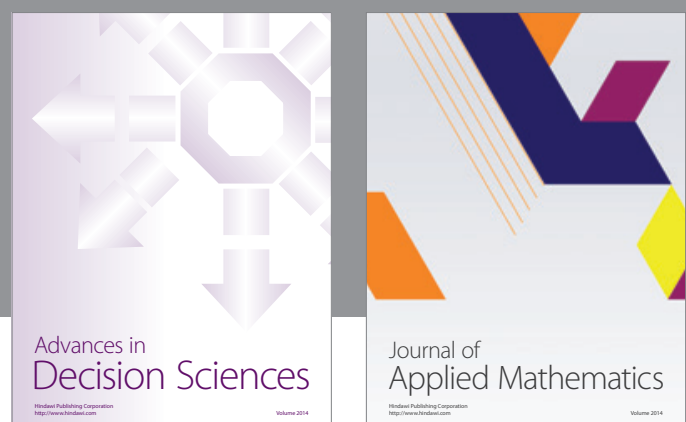

Journal of

Applied Mathematics
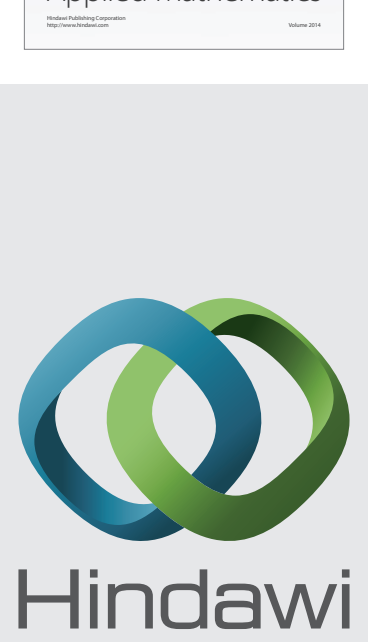

Submit your manuscripts at http://www.hindawi.com
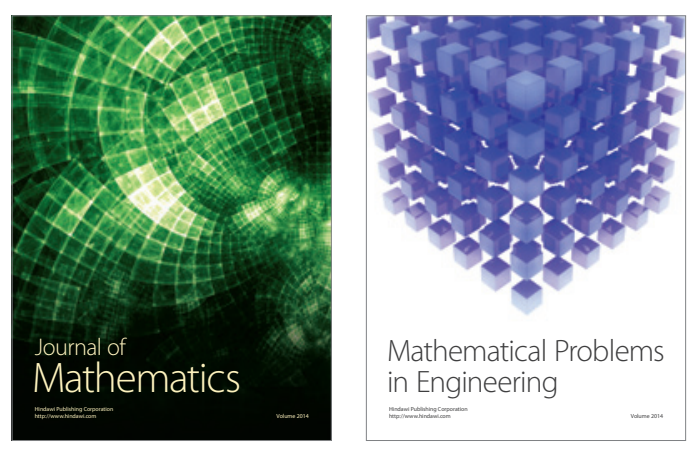

Mathematical Problems in Engineering
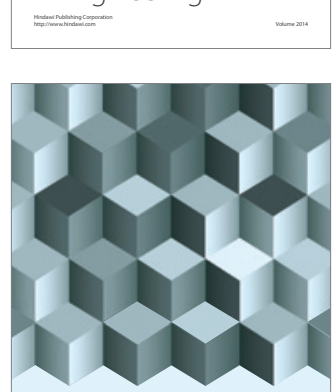

Journal of

Function Spaces
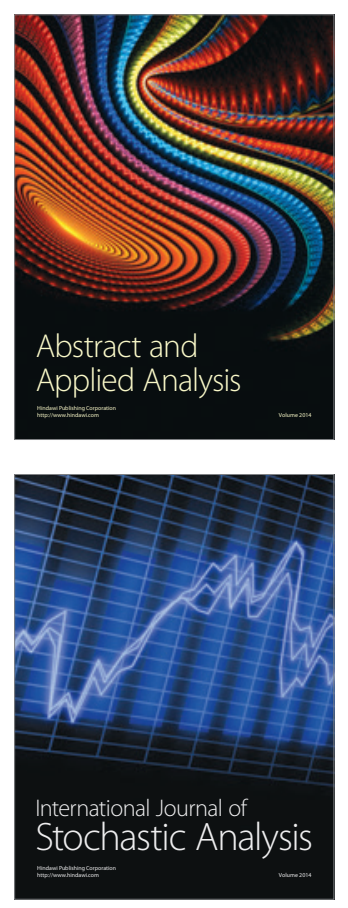

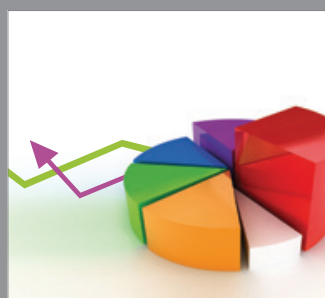

ournal of

Probability and Statistics

Promensencen
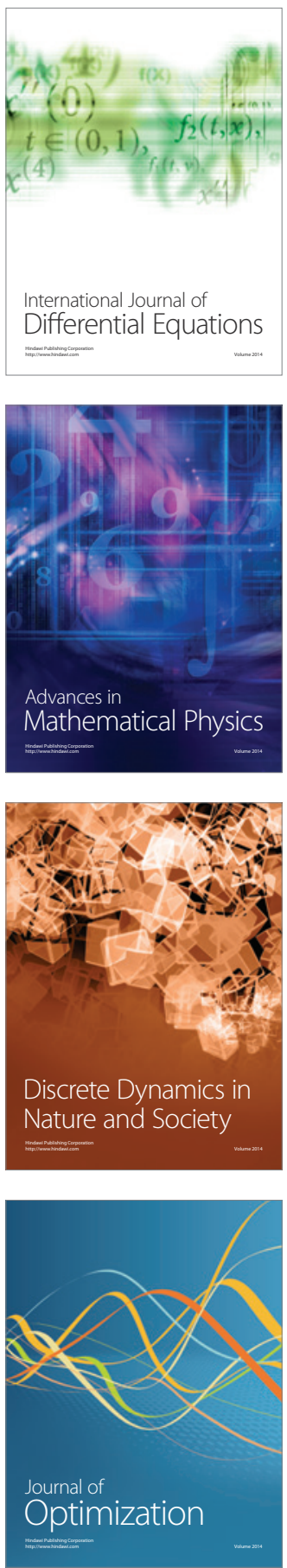